TRANSACTIONS OF THE

AMERICAN MATHEMATICAL SOCIETY

Volume 362, Number 1, January 2010, Pages 345-355

S 0002-9947(09)04799-0

Article electronically published on August 17, 2009

\title{
STRONG UNIQUE CONTINUATION FOR PRODUCTS OF ELLIPTIC OPERATORS OF SECOND ORDER
}

\author{
FERRUCCIO COLOMBINI AND HERBERT KOCH
}

\begin{abstract}
We study strong unique continuation for products of elliptic operators. The main tools are Carleman inequalities for second order elliptic operators. We obtain strong unique continuation assuming either Gevrey regularity of the coefficients or some pointwise conditions on the coefficients.
\end{abstract}

\section{INTRODUCTION}

Solutions to the scalar second order differential inequalities

$$
\left|\sum_{i, j=1}^{n} a^{i j}(x) \partial_{i j}^{2} u\right| \leq c(|u|+|\nabla u|)
$$

have the Strong Unique Continuation Property if the coefficients $a^{i j}$ are real, uniformly positive definite, and Lipschitz continuous: If $u$ satisfies (1), if it is flat at the origin in the sense that

$$
\sup _{r \leq \delta} r^{-N}\|u\|_{L^{2}(B(0, r))}<\infty
$$

for all $N$, then $u$ vanishes near the origin.

Building on the seminal work by Carleman [5], N. Aronszajn, A. Krzywicki, and J. Szarski [3] have obtained this basic result using Carleman inequalities. By now the understanding of the scalar second order case is fairly complete, and we refer to [10] for a more detailed exposition of its history.

In this note we study conditions on the differential equations

$$
P u=\sum_{|\beta| \leq 2 m} c_{\beta}(x) \partial^{\beta} u
$$

which ensure strong unique continuation. For simplicity we consider products of second order operators $P=A_{M} \ldots A_{1}$, where (using the Einstein convention)

$$
A_{j} u=a_{k l}^{j}(x) \partial_{k l}^{2} u \text {. }
$$

We assume that the coefficients $a_{k l}^{j}$ are in the Gevrey class $G^{\sigma}$ (see Section 2) and that there exists $\alpha>0$ such that the eigenvalues $\nu_{1} \leq \nu_{2} \cdots \leq \nu_{n}$ of $a_{k l}^{j}(x)$ satisfy

$$
\alpha>\frac{\nu_{n}-\nu_{1}}{\nu_{1}}
$$

uniformly in $x$ and $j$.

Received by the editors September 21, 2007 and, in revised form, January 15, 2008 2000 Mathematics Subject Classification. Primary 35B60.

(C)2009 American Mathematical Society Reverts to public domain 28 years from publication 
Theorem 1. Suppose that the operators $A_{k}$ and $\alpha$ satisfy (3) at $x=0$ (where we replace $A$ by $A_{k}$ ). Let $\sigma<1+1 / \alpha$. Then the Strong Unique Continuation Property holds for the differential equation

$$
P u=\sum_{|\beta| \leq[3 M / 2]} a_{\beta} \partial^{\beta} u
$$

provided the coefficients are in the Gevrey class $G^{\sigma}$.

The case of higher order operators is very different from the situation in (1). In general strong unique continuation does not hold (see Alinhac [1); however a strengthened flatness assumption like (4) below sometimes implies vanishing.

The proof of the theorem consists of two parts. First Gevrey regularity of the elliptic equation implies Gevrey regularity of the solution (see Lerner [12] and Section 2). Together with the vanishing of infinite order this implies

$$
|u| \lesssim e^{-|x|^{-s}} \text { near } x=0 \text { and for some } s>0 .
$$

In a second step Carleman inequalities show that (4) implies vanishing near the origin.

The situation improves for powers of the Laplacian, where one can prove Carleman inequalities by applying more standard Carleman inequalities to each factor. This line of approach has been taken in [6] and [2] for the differential inequalities

$$
\left|\Delta^{m} u\right| \leq \sum_{j=0}^{m} c_{j}|x|^{j-2 m}\left|D^{j} u\right|
$$

for $c_{m}$ sufficiently small. It has been improved by [11] for $m=2$ to the same conclusion for the inequality

$$
\left|\Delta^{2} u\right| \leq c_{0}|x|^{-4}|u|+c_{1}|x|^{-3}|D u|+c_{2}|x|^{-2}\left|D^{2} u\right|+c_{3}|x|^{-1+\varepsilon}\left|D^{3} u\right|,
$$

where again $c_{2}$ is small.

In this note we generalize the result of $[8$ to operators of all orders, and we allow derivatives on the right hand side. We also improve upon the $\varepsilon$ in (5) and generalize the result of [11] to products of elliptic operators of second order for which $a_{k l}^{j}(0)=\delta_{k l}$. At the same time we provide a unified proof for Theorem 1 and for the generalization of [11.

Let $u$ satisfy a differential equality or inequality. Weak unique continuation is the implication: Vanishing in an open set implies vanishing. The book of Zuily 14. gives an interesting and detailed survey of this theory; it is instructive but beyond the scope of this paper to compare the results on strong and weak unique continuation. In view of Watanabe 13 one might conjecture that solutions to

$$
\left|\Delta^{3} u+\sum_{|\alpha|=5} c_{\alpha} \partial^{\alpha} u\right| \leq \sum_{j \leq 4} c_{j}\left|D^{j} u\right|
$$

with Lipschitz continuous functions $c_{\alpha}$ have the strong unique continuation property.

The paper at hand implements the procedure above in a fairly straightforward manner. All constructions rely on the following observations: The weight $|x|^{\tau}$ is only degenerate pseudoconvex. We replace it by $|x|^{h_{\tau}(-\ln |x|)}$ with convex functions $h_{\tau}$. Linear functions $h$ allow us to control one derivative for the second order operator and $M$ derivatives for a product of $M$ second order operators-provided 
the coefficients are $\delta^{i j}$ at $x=0$. Convexity of $h$ allows us to control perturbations from the Laplacian and $M / 2$ more derivatives. The amount of convexity of $h$ however is limited by the at most linear growth translating to polynomial vanishing for $u$. We may use more convexity at the expense of requiring more decay as in (4).

\section{Gevrey Regularity for elliptic operators}

Definition 2.1. Let $U$ be an open set, $f \in C^{\infty}(U)$, and $\sigma>1$. We say that $f$ lies in the Gevrey class $\mathcal{G}^{\sigma}$ if there exist constants $c$ and $A$ so that

$$
\left|\partial^{\alpha} f\right| \leq c A^{|\alpha|}|\alpha| !^{\sigma} \quad \text { in } U .
$$

It is not hard to see that we get the same space if we replace $|\alpha|^{\sigma}$ by $(\alpha !)^{\sigma}$ or by $|\alpha|^{\sigma|\alpha|}$.

Lemma 2.2. Suppose that $0 \in U, \sigma \geq 1$, and that $f \in \mathcal{G}^{\sigma}(U)$ satisfies

$$
\partial^{\alpha} f(0)=0 \quad \text { for all multiindices } \alpha .
$$

Let $\sigma-1<\rho$. Then

$$
|f(x)| \leq e^{-|x|^{-1 / \rho}}
$$

near $x=0$.

This estimate follows from Taylor's formula by optimizing the order. See 12 for a complete proof. This decay result is fairly sharp because the following example can be checked by a straightforward calculation, which again can be found in [12].

Lemma 2.3. We have

$$
e^{-|x|^{-1 / \rho}} \in \mathcal{G}^{\sigma}(\mathbb{R})
$$

provided $1+\rho=\sigma$.

Gevrey classes are good classes for elliptic regularity; see [4], Proposition 2.13:

Lemma 2.4. Let

$$
P(x, D) u=f \quad \text { in } U
$$

be an elliptic differential equation with coefficients and right hand side in the Gevrey class $\mathcal{G}^{\sigma}(U)$. Then $u \in G^{\sigma}(V)$ for all bounded $V \subset \subset U$.

\section{Carleman inequalities For the Weight $e^{\tau|x|^{-\alpha}}$ FOR ELLIPTIC OPERATORS OF SECOND ORDER}

Let

$$
A=\sum_{i, j=1}^{n} a^{i j}(x) \partial_{i} \partial_{j}
$$

be an elliptic operator with real Lipschitz continuous coefficients. The following Carleman inequality has been proven in [8]:

Proposition 3.1. Suppose that the coefficients of $A$ are constant and positive definite, and denote the eigenvalues by $\nu_{1} \leq \nu_{2} \cdots \leq \nu_{n}$. We assume that $\alpha>0$ in (3). There exist $\tau_{0}>0$ and $r_{0}>0$ such that for $\tau>\tau_{0}$ the following inequality holds for all smooth $v$ supported in $B_{r_{0}}(0) \backslash\{0\}$ :

$$
\tau^{-1 / 2}\left\|D^{2}\left(|x|^{\alpha / 2} e^{\tau|x|^{-\alpha}} v\right)\right\|_{L^{2}}+\tau^{3 / 2}\left\||x|^{-2-3 \alpha / 2} e^{\tau|x|^{-\alpha}} v\right\|_{L^{2}} \lesssim\left\|e^{\tau|x|^{-\alpha}} A v\right\|_{L^{2}} .
$$


Its most interesting feature is the sharp inequality (3). Checking the proof, it is not hard to see that the inequality remains true for operators

$$
A v=\sum_{i, j} \partial_{i} a^{i j}(x) \partial_{j}
$$

with uniformly elliptic Lipschitz coefficients which satisfy (3) near $x=0$ and for functions supported in a small punctured disc.

We easily obtain a slightly stronger consequence: Under the same assumptions the estimate

$$
\sum_{j=0}^{2} \tau^{3 / 2-j}\left\||x|^{\alpha / 2}|x|^{(j-2)(1+\alpha)} D^{j} e^{\tau|x|^{-\alpha}} v\right\|_{L^{2}} \lesssim\left\|e^{\tau|x|^{-\alpha}} A v\right\|_{L^{2}}
$$

holds. To see this, we set $w=e^{\tau|x|^{-\alpha}} v$ and write

$$
|x|^{\alpha / 2} D^{2} w=D^{2}\left(|x|^{\alpha / 2} w\right)+O\left(|x|^{\alpha / 2-1}\right) D w+O\left(|x|^{\alpha / 2-2}\right) w,
$$

thus

$$
\left\||x|^{\alpha / 2} D^{2} w\right\|_{L^{2}} \leq\left\|D^{2}\left(|x|^{\alpha / 2} w\right)\right\|_{L^{2}}+c\left\||x|^{\alpha / 2-2} w\right\|_{L^{2}}+c\left\||x|^{\alpha / 2-1} D w\right\|_{L^{2}},
$$

and by integration by parts for the first order derivatives

$$
\left\||x|^{\alpha / 2} D^{2} w\right\|_{L^{2}} \leq\left\|D^{2}\left(|x|^{\alpha / 2} w\right)\right\|_{L^{2}}+c\left\||x|^{\alpha / 2-2} w\right\|_{L^{2}} .
$$

We combine this with the estimate of Proposition 3.1 to get

$$
\tau^{-1 / 2}\left\||x|^{\alpha / 2} D^{2} w\right\|_{L^{2}}+\tau^{3 / 2}\left\||x|^{-2-3 \alpha / 2} w\right\|_{L^{2}} \lesssim\left\|e^{\tau|x|^{-\alpha}} A v\right\|_{L^{2}} .
$$

We can sharpen the estimate from the integration by parts to

$$
\begin{aligned}
\tau^{1 / 2}\left\||x|^{\alpha / 2}|x|^{-1-\alpha} D w\right\|_{L^{2}} \leq & C\left(\tau^{3 / 2}\left\||x|^{\alpha / 2}|x|^{-2(1+\alpha)} w\right\|_{L^{2}}\right. \\
& \left.+\tau^{-1 / 2}\left\||x|^{\alpha / 2} D^{2} w\right\|_{L^{2}}\right),
\end{aligned}
$$

which completes the proof of inequality (6).

It will be useful in the sequel to commute the derivatives and the exponential. We compute

$$
|x|^{\alpha / 2}|x|^{-1-\alpha} e^{\tau|x|^{-\alpha}} D v=|x|^{\alpha / 2}|x|^{-1-\alpha} D w+\alpha \tau|x|^{\alpha / 2}|x|^{-2-2 \alpha}|x|^{-1} w x
$$

and hence

$$
\tau^{1 / 2}\left\||x|^{\alpha}|x|^{-1-\alpha} e^{\tau|x|^{-\alpha}} D v\right\|_{L^{2}} \lesssim\left\|e^{\tau|x|^{-\alpha}} A v\right\|_{L^{2}}
$$

Similarly we can deal with the second order derivative. We obtain the Carleman inequality in the equivalent form

$$
\sum_{j=0}^{2} \tau^{3 / 2-j}\left\|e^{\tau|x|^{-\alpha}}|x|^{\alpha / 2}|x|^{(j-2)(1+\alpha)} D^{j} v\right\|_{L^{2}} \lesssim\left\|e^{\tau|x|^{-\alpha}} A v\right\|_{L^{2}} .
$$

In the sequel we also need higher order derivatives and different powers in the estimate.

Corollary 3.2. Let

$$
A u=\sum_{i, j} \partial_{i} a^{i j}(x) \partial_{j} u
$$


with $a^{i j}$ positive definite and continuous. Suppose that (3) holds at $x=0$. Let $\sigma>0$, and assume that the coefficients are of class $C^{\sigma}$. Then there exists $\tau_{0}$ such that for all $|s|, k \leq \sigma$, and $\tau \geq \tau_{0}$

$$
\begin{aligned}
\sum_{j=0}^{k+2} \tau^{3 / 2-j} & \left\||x|^{\alpha / 2+j(1+\alpha)}|x|^{s} e^{\tau|x|^{-\alpha}} D^{j} v\right\|_{L^{2}} \\
& \lesssim \sum_{j=0}^{k} \tau^{-j}\left\||x|^{(2+j)(1+\alpha)}|x|^{s} e^{\tau|x|^{-\alpha}} D^{j} A v\right\|_{L^{2}} .
\end{aligned}
$$

Proof. We apply estimate (8) to the functions $u=|x|^{s+(2+k)(1+\alpha)} \partial^{\beta} v$ with $|\beta|=k$, and we obtain

$$
\begin{gathered}
\sum_{j=0}^{2} \tau^{3 / 2-j-k}\left\||x|^{\alpha / 2}|x|^{(j-2)(1+\alpha)} e^{\tau|x|^{-\alpha}} D^{j}\left(|x|^{s+(2+k)(1+\alpha)} \partial^{\beta} v\right)\right\|_{L^{2}} \\
\lesssim \tau^{-k}\left\|e^{\tau|x|^{-\alpha}} A|x|^{s+(2+k)(1+\alpha)} \partial^{\beta} v\right\|_{L^{2}} .
\end{gathered}
$$

We sum over $\beta$ with $|\beta|=k$. On the right hand side we commute $A$ and $|x|^{s+(2+k)(1+\alpha)} D^{k}$, which yields commutator terms which are controlled by the left hand side. On the left hand side we commute $D^{j}$ and $|x|^{s+(2+k)(1+\alpha)}$, where the commutator terms are controlled by induction on $k$.

\section{Products of Elliptic operators}

We study

$$
P=A_{M} \ldots A_{1} .
$$

Let $\left(\nu_{j k}\right)_{1 \leq j \leq n}$ be the eigenvalues of the coefficients of the $k$ th operator at $x=0$. We assume that (3) is satisfied for all $j$. Corollary 3.2 is applied iteratively. Let $v_{l}=A_{l} \ldots A_{1} v$. Then

$$
\begin{aligned}
\sum_{j=0}^{2 M} \tau^{3 M / 2-j} & \left\||x|^{M(-2-3 \alpha / 2)}|x|^{j(1+\alpha)} e^{\tau|x|^{-\alpha}} D^{j} v\right\|_{L^{2}} \\
& \lesssim \sum_{j=0}^{2 M-2} \tau^{3(M-1) / 2-j}\left\||x|^{(1-M)(2+3 \alpha / 2)}|x|^{j(1+\alpha)} e^{\tau|x|^{-\alpha}} D^{j} v_{1}\right\|_{L^{2}}
\end{aligned}
$$

by Corollary 3.2 with $k=2(M-1)$ and $s=-M\left(2+\frac{3}{2} \alpha\right)-\alpha / 2$

$$
\lesssim \sum_{j=0}^{4} \tau^{3-j}|||x|^{-4-3 \alpha}|x|^{j(1+\alpha)} e^{\tau|x|^{-\alpha}} D^{j} v_{M-2} \|
$$

after $M-1$ applications of Corollary 3.2

$$
\lesssim \sum_{j=0}^{2} \tau^{3 / 2-j}|||x|^{-2-3 \alpha / 2}|x|^{j(1+\alpha)} e^{\tau|x|^{-\alpha}} D^{j} v_{M-1} \|
$$

by Corollary 3.2 with $k=2$ and $s=-4-7 \alpha / 2$

$$
\lesssim\left\|e^{\tau|x|^{-\alpha}} v_{M}\right\|
$$

by Corollary 3.2 with $k=0$ and $s=-2(1+\alpha)$. 
Proof of Theorem 1: The operator is hypoelliptic in the Gevrey class $\sigma$. Thus vanishing of infinite order implies that

$$
|u| \lesssim e^{-|x|^{-\beta}}
$$

for some $\beta>\alpha$ and small arguments. Now the Carleman inequality (10) implies vanishing by standard arguments.

A related result about weak unique continuation can be found in Zuily [14, Theorem 2.5.1.

\section{Products of Laplacians}

Here we study products of operators

$$
A_{l}=a_{l}^{i j} \partial_{i j}^{2}
$$

with

$$
a_{l}^{i j}(0)=\delta^{i j}
$$

and

$$
a_{l}^{i j} \in C^{2(M-l)}
$$

for $1 \leq l \leq M$. We study again the Strong Unique Continuation Property, but this time we want to assume only vanishing of infinite order and not the stronger exponential bound.

Theorem 2. Suppose that the operators $A_{l}$ satisfy (11). Let $C>0$. Then there exists $\delta>0$ such that the Strong Unique Continuation Property holds for the differential inequality

$$
\begin{aligned}
\left|A_{M} A_{M-1} \ldots A_{1} u\right| \leq & \sum_{j=0}^{M-1} C|x|^{j-2 m}\left|D^{j} u\right|+\delta|x|^{-M}\left|D^{M} u\right| \\
& +C \sum_{j=M+1}^{[3 M / 2]}|\ln | x||^{M-j-\varepsilon}|x|^{j-2 m}\left|D^{j} u\right|
\end{aligned}
$$

if $\varepsilon>0$.

Given $1 \ll \tau$, we use weight functions $e^{h(-\ln (|x|))}$ with

$$
\tau \sim h^{\prime}
$$

i.e., there exists $C>1$ with $C^{-1} \tau \leq h^{\prime} \leq C \tau$,

$$
\begin{gathered}
\frac{1}{4} \leq h^{\prime \prime}+d\left(2 h^{\prime}, \mathbb{Z}\right), \\
\left|h^{\prime \prime \prime}\right| \lesssim\left(h^{\prime \prime}+1\right) \lesssim \tau .
\end{gathered}
$$

Such functions are used to dominate error terms in the Carleman inequalities. They can be constructed as follows. Let $\left(c_{j}\right)_{j \in \mathbb{Z}}$ be a sequence of nonnegative numbers with

$$
\sum c_{j}<\delta
$$

for a small number $\delta$ which will be independent of $\tau$. Let $\left(a_{j}\right)$ be the discrete convolution of $\left(c_{j}\right)$ and $2^{-\mu|j|}$ for some small $\mu>0$. Then $c_{j} \leq a_{j}$ and $2^{-\mu} a_{j+1} \leq$ $a_{j} \leq 2^{\mu} a_{j+1}$ for all $j$, a property which we call slowly varying. We follow the construction of Lemma 6.1 of [10] which deals with a slightly more complicated 
situation. We define a function $h$ with $h(0)=0, h^{\prime}(-\infty)=[\tau]+\frac{5}{4}$ ([.] the largest integer smaller than $\tau$ ),

$$
h^{\prime \prime}=\sum b_{j} \chi_{[j, j+1]}
$$

where the $b_{j}$ are integers satisfying

$$
\begin{aligned}
b_{j}=0 & \text { if } a_{j} \leq \mu \tau^{-1}, \\
b_{j} \in\left[\tau a_{j}, 2 \tau a_{j}\right] & \text { if } a_{j} \geq \mu \tau^{-1} .
\end{aligned}
$$

This function $h$ has almost all the desired properties if $\delta$ and $\mu$ are sufficiently small, and we obtain the missing statement (15) by a standard mollification.

Proposition 5.1. Suppose that $A$ is a second order elliptic operator as above with Lipschitz continuous coefficients, and let $1 \ll \tau$. Then there exists $h$ which satisfies (13), (14), (15) and

$$
\sum_{j=0}^{2} \tau^{1-j}\left\|\left(1+h^{\prime \prime}\right)^{\frac{1}{2}}|x|^{-2+j} e^{h} D^{j} v\right\| \lesssim\left\|e^{h} A v\right\|
$$

for all $v$ supported in $B(0,1) \backslash\{0\}$ with a constant in inequality (16) independent of $\tau$ and $h$.

Proof. The result is contained in 10, and it is a small modification of classical arguments. Since the argument is elementary, we include it for completeness, while explicitly disclaiming originality. We may assume that $|x|^{-1}\left(1+h^{\prime \prime}(-\ln (|x|))^{\frac{1}{2}}\right)$ is large. Then first order terms can be controlled, and it suffices to prove the estimate for operators in divergence form, $A u=\partial_{i}\left(a^{i j}(x) \partial_{j} u\right)$.

We prove the estimate parallel for the Laplace operator and in the general case. Let $t=-\ln |x|, y=x /|x| \in \mathbb{S}^{n-1}$. Then

$$
|x|^{\frac{n+2}{2}} \Delta|x|^{\frac{2-n}{2}}=\partial_{t}^{2}+\Delta_{\mathbb{S}^{n-1}}-\left(\frac{n-2}{2}\right)^{2}=L_{0},
$$

and, in symbolic notation,

$$
L=|x|^{\frac{n+2}{2}} A|x|^{\frac{2-n}{2}}=L_{0}+D b D+D c_{1}+c_{2} D+d,
$$

where the operator on the right hand side is expressed in the $\mathbb{S}^{n-1} \times \mathbb{R}$ coordinates. The coefficients $b, c_{1}, c_{2}$, and $d$ are bounded by $C_{0} e^{-t}$.

We recall that by the chain rule $\sum_{j=0}^{l}|x|^{j}\left|D_{x}^{j} u\right| \sim \sum_{j=0}^{l}\left|D_{t, y}^{j} u\right|$, and the Jacobian determinant leads to

$$
\int f d x=\int_{\mathbb{R}} \int_{\mathbb{S}^{n-1}} e^{n t} f d t d y .
$$

Thus the desired inequality is equivalent (modulo adding the half of an integer to $\left.h^{\prime}\right)$ to

$$
\sum_{j=0}^{2} \tau^{1-j}\left\|\left(1+h^{\prime \prime}\right)^{1 / 2} e^{h(t)} D_{t, y}^{j} v\right\|_{L^{2}\left(\mathbb{R} \times \mathbb{S}^{n-1}\right)} \leq\left\|e^{h(t)} L v\right\|_{L^{2}\left(\mathbb{R} \times \mathbb{S}^{n-1}\right)}
$$

and, repeating previous arguments and using $h^{(j)} \lesssim \tau$ for $j=1,2$, to

$$
\sum_{j=0}^{2} \tau^{1-j}\left\|\left(1+h^{\prime \prime}\right)^{1 / 2} D_{t, y}^{j} v\right\|_{L^{2}\left(\mathbb{R} \times \mathbb{S}^{n-1}\right)} \leq\left\|L_{h} v\right\|_{L^{2}\left(\mathbb{R} \times \mathbb{S}^{n-1}\right)}
$$


where $L_{h}$ is the conjugated operator

$$
L_{h}=e^{h} L e^{-h} .
$$

For simplicity we do the calculation first for $L_{0}$ :

$$
L_{0, h}=e^{h} L_{0} e^{-h}=L_{0}+\left(h^{\prime}\right)^{2}-\left(\partial_{t} h^{\prime}+h^{\prime} \partial_{t}\right) .
$$

We denote $L_{0, h}^{s}=L_{0}+\left(h^{\prime}\right)^{2}$ and $L_{0, h}^{u}=-\left(\partial_{t} h^{\prime}+h^{\prime} \partial_{t}\right)$. Then

$$
\left\|L_{0, h} v\right\|_{L^{2}}^{2}=\left\|L_{0, h}^{s} v\right\|_{2}^{2}+\left\|\left(\partial_{t} h^{\prime}+h^{\prime} \partial_{t}\right) v\right\|^{2}-\left(\left[L_{0, h}^{u}, L_{0, h}^{s}\right] u, u\right)
$$

where

$$
\left(\left[L_{0, h}^{u}, L_{0, h}^{s}\right] u, u\right)=-4 \int h^{\prime} h^{\prime \prime} h^{\prime} u^{2}+h^{\prime \prime}\left(\partial_{t} u\right)^{2}-h^{(4)} u^{2} d y d t .
$$

The last term is negligible. We obtain the desired estimate for $u$ in the case of the Laplacian. First and second order terms are controlled by elliptic estimates on annuli using the bounds for $u$ and $\left\|L_{0, h}^{s} u\right\|_{L^{2}}^{2}$.

The calculation for $L$ is similar. We again decompose $L_{h}$ into a symmetric and an antisymmetric part. The commutator contains additional error terms compared to $L_{0, h}$, which can be controlled by

$$
C \int_{\mathbb{R}} \int_{\mathbb{S}^{n-1}} e^{-t}\left(|D u|^{2}+\tau^{2}|u|^{2}\right) d t d \sigma .
$$

This term can be controlled by the left hand side of (16) if

$$
h^{\prime \prime}+\frac{1}{4} \geq 2 C \tau e^{-t}
$$

as long as the coefficients $b, c_{1}, c_{2}, d$ and their derivatives are small. We choose $h$ with

$$
\begin{gathered}
h^{\prime}(t)=[\tau]+\frac{1}{4} \quad \text { if } t \geq 2 C \ln \tau, \\
h^{\prime \prime}(t)=2 C \tau e^{-t} \quad \text { if } 0 \leq t \leq C \ln \tau .
\end{gathered}
$$

With this argument we obtain the estimate provided $u$ is supported in a small punctured disc with size independent of $\tau$. The general estimate follows using arguments as in Hörmander, 9] Chapter 17.2.

Corollary 5.2. Let $\sigma>0$ and suppose that the coefficients are $\sigma+1$ times continuously differentiable. Then there exists $\tau_{0}$ such that for all $|s|, k \leq \sigma$, and $\tau \geq \tau_{0}$ the estimate

$$
\sum_{j=0}^{k+2} \tau^{1-j}\left\|\left(1+h^{\prime \prime}\right)^{\frac{1}{2}}|x|^{j+s} e^{h} D^{j} v\right\| \lesssim \sum_{j=0}^{k} \tau^{-j}\left\||x|^{2+j+s} e^{h} D^{j} A v\right\|
$$

holds.

Proof. We argue as in the previous section. There is no difference in the argument.

Again we apply this iteratively.

Proposition 5.3. Suppose that $A_{l}, 1 \leq l \leq M$, are elliptic operators of second order with coefficients in $C^{1+2(M-l)}$ with $a_{l}^{i j}(0)=\delta^{i j}$. Let

$$
P=A_{M} \ldots A_{1} \text {. }
$$


Then the following Carleman inequality holds for $\tau$ sufficiently large and $v \in$ $C_{0}^{\infty}\left(B_{1}(0) \backslash\{0\}\right)$ :

$$
\sum_{j=0}^{2 M} \tau^{M-j}\left\|\left(1+h^{\prime \prime}\right)^{M / 2}|x|^{-2 M+j} e^{h} D^{j} v\right\|_{L^{2}} \lesssim\left\|e^{h} P v\right\| .
$$

Proof. Again we define for $1 \leq j \leq M$

$$
v_{j}=A_{j} A_{j-1} \ldots A_{1} v \text {. }
$$

We apply Corollary 5.2 iteratively:

$$
\begin{aligned}
\sum_{j=0}^{2 M} \tau^{M-j} \|(1+ & \left.h^{\prime \prime}\right)^{M / 2}|x|^{-2 M+j} e^{h} D^{j} v \|_{L^{2}} \\
& \lesssim \sum_{j=0}^{2 M-2} \tau^{M-1-j}\left\|\left(1+h^{\prime \prime}\right)^{(M-1) / 2}|x|^{-2(M-1)+j} e^{h} D^{j} v_{1}\right\|_{L^{2}} \\
& \lesssim \sum_{j=0}^{4} \tau^{2-j}\left\|\left(1+\left|h^{\prime \prime}\right|\right)|x|^{-4+j} e^{h} D^{j} v_{M-2}\right\| \\
& \lesssim \sum_{j=0}^{2} \tau^{1-j}\left\|\left(1+h^{\prime \prime}\right)^{1 / 2}|x|^{-2+j} e^{h} D^{j} v_{M-1}\right\| \\
& \lesssim\left\|e^{h} v_{M}\right\| .
\end{aligned}
$$

We study strong unique continuation for solutions to the inequality

$$
|P v| \leq \sum_{j=0}^{3 M / 2} b_{j}(x)|x|^{-2 M+j}\left|D^{j} v\right| .
$$

We have to choose $h$ such that

$$
b_{j} \lesssim \tau^{M-j}\left(1+h^{\prime \prime}\right)^{M / 2} .
$$

This reduces to boundedness for $j<M$ and for $j=M$ to

$$
\limsup _{x \rightarrow 0} b_{M}(x) \ll 1
$$

with an implicit constant $C$ depending only on $h$ and $n$. For all $j$ we need

$$
h^{\prime \prime} \geq \max \left\{\left(\sum_{j=M+1}^{[3 M / 2]} b_{j} \tau^{j-M}\right)^{2 / M}-1,0\right\}
$$

and

$$
\int_{1}^{\infty} h^{\prime \prime}(s) d s \lesssim \tau .
$$

This is satisfied under the assumptions of Theorem 2, The Strong Unique Continuation Property follows again by standard arguments.

Let $c_{j, k}=\max \left\{b_{j}(x): 2^{-k} \leq|x| \leq 2^{-k+1}\right\}$. Thus we need

$$
\sum_{k} c_{j, k}^{2 / M} \lesssim \tau^{3-2 j / M}
$$


where we sum only over those $k$ for which

$$
c_{j, k} \geq \tau^{M-j}
$$

We work out these condition for $M=2,3,4$.

The case $M=2$. In this case we consider a fourth order operator. It suffices to assume that $b_{0}$ and $b_{1}$ are large constants, $b_{2}$ is a small constant, and the coefficients $c_{3, k}$ are summable.

The case $M=3$. Here it suffices to require that $b_{0}, b_{1}$, and $b_{2}$ be large constants. The coefficent $b_{3}$ of the third order terms has to be small. Since $5>3 * 3 / 2$, we require $b_{5}=0$, and for $b_{4}$ we obtain the condition

$$
\limsup _{\lambda \rightarrow 0} \lambda^{1 / 3} \sum_{k: c_{4, k}^{2 / 3} \geq \lambda} c_{4, k}^{2 / 3} \ll 1 .
$$

Lemma 5.4. Let $\left(a_{j}\right) \in l^{p}$. Then

$$
\sup _{\lambda>0} \lambda^{p-1} \sum_{\left|a_{j}\right| \geq \lambda}\left|a_{j}\right| \lesssim\left\|\left(a_{j}\right)\right\|_{l^{p}}^{p}
$$

Proof. By Hölder's and Tschebysheff's inequalities

$$
\sum_{\left|a_{j}\right| \geq \lambda}\left|a_{j}\right| \leq\left\|\left(a_{j}\right)\right\|_{l^{p}}\left(\#\left\{j:\left|a_{j}\right| \geq \lambda\right\}\right)^{1-1 / p} \leq \lambda^{1-p}\left\|\left(a_{j}\right)\right\|_{l^{p}}^{p} .
$$

Thus (21) is satisfied if

$$
\left(c_{4, k}\right) \in l^{1}
$$

The case $M=4$. Here we need that $b_{0}, b_{1}, b_{2}$, and $b_{3}$ are arbitrary constants, $b_{4}$ is a small constant, $b_{7}=0$,

$$
\sum_{k} c_{6, k}^{1 / 2}<\infty
$$

and

$$
\sum_{k} c_{5, k}<\infty
$$

The case $M$ even. We need $b_{j}$ bounded for $j<M, b_{M} \ll 1, b_{j}=0$ for $j>3 M / 2$,

$$
\sum_{k} c_{3 M / 2, k}^{2 / M}<\infty
$$

and, if $M \geq 6$,

$$
\sum_{k: c_{3 M / 2-1, k} \geq \tau^{1-M / 2}} c_{3 M / 2-1, k}^{2 / M} \lesssim \tau^{2 / M}
$$

which is satisfied if $\left(c_{3 M / 2-1, k}\right) \in l^{\frac{2}{M-2}}$.

In general we obtain the sufficient condition

$$
\left(c_{M+j, k}\right) \in l^{\frac{1}{j}}
$$

for $0<j \leq \frac{M}{2}$. 


\section{REFERENCES}

[1] S. Alinhac. Non-unicité pour des opérateurs différentiels à caractéristiques complexes simples. Ann. Sci. École Norm. Sup. (4), 13(3):385-393, 1980. MR:597745 (83b:35004b)

[2] S. Alinhac and M. S. Baouendi. Uniqueness for the characteristic Cauchy problem and strong unique continuation for higher order partial differential inequalities. Amer. J. Math., 102(1):179-217, 1980. MR:556891 (81e:35003)

[3] N. Aronszajn, A. Krzywicki, and J. Szarski. A unique continuation theorem for exterior differential forms on Riemannian manifolds. Ark. Mat., 4:417-453, 1962. MR0140031 (25:3455)

[4] L. Boutet de Monvel and P. Krée. Pseudo-differential operators and Gevrey classes. Ann. Inst. Fourier (Grenoble), 17(fasc. 1):295-323, 1967. MR0226170

[5] T. Carleman. Sur un problème d'unicité pur les systèmes d'équations aux dérivées partielles à deux variables indépendantes. Ark. Mat. Astr. Fys., 26(17):9, 1939. MR0000334 (1:55f)

[6] F. Colombini and C. Grammatico. Some remarks on strong unique continuation for the Laplace operator and its powers. Comm. Partial Differential Equations, 24(5-6):1079-1094, 1999. MR:1680873(2000c:35263)

[7] F. Colombini and C. Grammatico. A result on strong uniqueness in Gevrey spaces for some elliptic operators. Comm. Partial Differential Equations, 30(1-3):39-57, 2005. MR2131044 (2006c:35025)

[8] Ferruccio Colombini, Cataldo Grammatico, and Daniel Tataru. Strong uniqueness for second order elliptic operators with Gevrey coefficients. Math. Res. Lett., 13(1):15-27, 2006. MR2199563 (2006j:35040)

[9] L. Hörmander. The analysis of linear partial differential operators. III, Springer-Verlag, 1985. MR781536

[10] Herbert Koch and Daniel Tataru. Carleman estimates and unique continuation for secondorder elliptic equations with nonsmooth coefficients. Comm. Pure Appl. Math., 54(3):339-360, 2001. MR1809741 (2001m:35075)

[11] Philippe Le Borgne. Unicité forte pour le produit de deux opérateurs elliptiques d'ordre 2. Indiana Univ. Math. J., 50(1):353-381, 2001. MR1857040 (2003i:35064)

[12] Nicolas Lerner. Résultats d'unicité forte pour des opérateurs elliptiques à coefficients Gevrey. Comm. Partial Differential Equations, 6, no. 10, 1163-1177, 1981. MR632767 (83m:35063)

[13] Kinji Watanabe. Sur l'unicité dans le problème de Cauchy pour les opérateurs différentiels à caractéristiques de multiplicité constante. Tôhoku Math. J. (2), 31(2):151-164, 1979. MR.538916 (80g:35005)

[14] Claude Zuily. Uniqueness and nonuniqueness in the Cauchy problem, volume 33 of Progress in Mathematics. Birkhäuser Boston Inc., Boston, MA, 1983. MR701544 (85d:35009)

Department of Mathematics, University of Pisa, Via F Buonarroti 2, 56127 Pisa, ITALY

Mathematisches Institut, University of Bonn, Beringstrasse 1, 53115 Bonn, Germany 